\title{
Methylase-assisted subcloning for high throughput BioBrick assembly
}

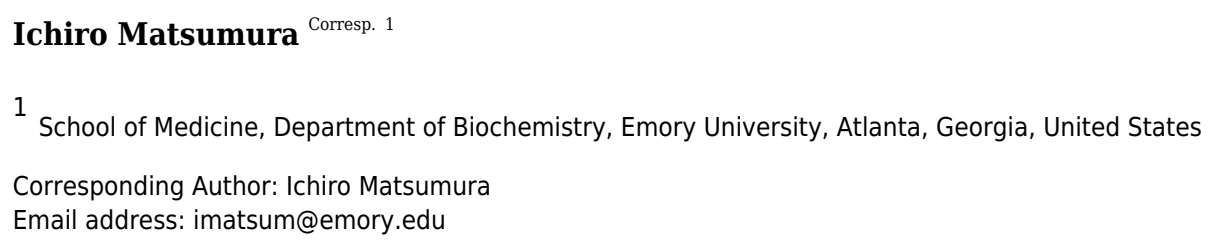

The BioBrick standard makes possible iterated pairwise assembly of cloned parts without any depletion of unique restriction sites. Every part that conforms to the standard is compatible with every other part, thereby fostering a worldwide user community. The assembly methods, however, are labor intensive or inefficient compared to some newer ones so the standard may be falling out of favor. An easier way to assemble BioBricks is described herein. Plasmids encoding BioBrick parts are purified from Escherichia coli cells that express a foreign site-specific DNA methyltransferase, so that each is subsequently protected in vitro from the activity of a particular restriction endonuclease. Each plasmid is double-digested and all resulting restriction fragments are ligated together without gel purification. The ligation products are subsequently double-digested with another pair of restriction endonucleases so only the desired insert-recipient vector construct retains the capacity to transform $E$. coli. This $4 \mathrm{R} / 2 \mathrm{M}$ BioBrick assembly protocol is more efficient and accurate than established workflows including $3 \mathrm{~A}$ assembly. It is also much easier than gel purification to miniaturize, automate and perform at high throughput. As such, it should streamline DNA assembly for the existing community of BioBrick users, and possibly encourage others to join. 
1 Manuscript for re-submission as an article to PeerJ, July 7, 2020

2

3

4

5

6

7 Methylase-assisted subcloning for high throughput BioBrick assembly

8

9 Ichiro Matsumura ${ }^{1}$

10

11

12

$13{ }^{1}$ Department of Biochemistry, Emory University School of Medicine, Atlanta, Georgia, USA

14 Corresponding author:

15 Ichiro Matsumura

$16 \quad 1510$ Clifton Road NE, room 4119

17 Atlanta, GA 30322, USA

18 e-mail address: imatsum@emory.edu

19 tele: (404) 727-5625

20

21

22

23 


\section{Abstract}

25

26

27

The BioBrick standard makes possible iterated pairwise assembly of cloned parts without any depletion of unique restriction sites. Every part that conforms to the standard is compatible with every other part, thereby fostering a worldwide user community. The assembly methods, however, are labor intensive or inefficient compared to some newer ones so the standard may be falling out of favor. An easier way to assemble BioBricks is described herein. Plasmids encoding BioBrick parts are purified from Escherichia coli cells that express a foreign site-specific DNA methyltransferase, so that each is subsequently protected in vitro from the activity of a particular restriction endonuclease. Each plasmid is double-digested and all resulting restriction fragments are ligated together without gel purification. The ligation products are subsequently doubledigested with another pair of restriction endonucleases so only the desired insert-recipient vector construct retains the capacity to transform $E$. coli. This $4 \mathrm{R} / 2 \mathrm{M}$ BioBrick assembly protocol is more efficient and accurate than established workflows including $3 \mathrm{~A}$ assembly. It is also much easier than gel purification to miniaturize, automate and perform at high throughput. As such, it should streamline DNA assembly for the existing community of BioBrick users, and possibly encourage others to join. 


\section{Introduction}

A bottleneck in many synthetic biology projects is the physical linkage of cloned synthetic genes ("parts") to each other to form longer functional assemblies (“devices"). The costs of gene synthesis, cloning and DNA sequencing have decreased significantly but syntheses are still limited in length $(\leq 3 \mathrm{~kb})$, nucleotide composition, accuracy and yield (Kosuri \& Church 2014; Kuhn et al. 2017). Many DNA assembly methods have been invented (Casini et al. 2015;

Chao et al. 2015; Sands \& Brent 2016; Vazquez-Vilar et al. 2018; Watson \& Garcia-Nafria 2019), which suggests that none work well for every user. The challenges of assembling cloned parts are not identical to those of ligating PCR products into plasmids (Bryksin \& Matsumura 2010) so different solutions are demanded.

Many synthetic biologists have adopted cloning standards that stipulate particular type II or type IIS restriction sites at the ends of each DNA "part." The BioBrick RCF[10] standard (Knight 2003) is most established (Figure 1). All BioBrick-compliant plasmids contain a characteristic pattern of sites recognized by type II restriction endonucleases (EcoRI-NotI-XbaIinsert-SpeI-NotI-PstI). Two such inserts can be combined by digesting one plasmid (recipient) with SpeI and PstI, and the other (donor) with XbaI and PstI. Alternatively, one plasmid (recipient) can be cut with EcoRI and XbaI, and the other with EcoRI and SpeI (donor). The overhangs of XbaI and SpeI digests products are compatible but anneal to form a "scar" not recognized by either restriction endonuclease. The ligation of the desired insert to the desired recipient plasmid thus creates a new BioBrick-compatible plasmid. The virtue of this approach compared to ad hoc subcloning strategies is that an infinite number of inserts can be combined, two at a time, without running out of unique restriction sites. The problem, and focus of this 
68 study, is that conventional subcloning (Matsumura 2015), particularly the gel purification step,

69 remains labor-intensive and recalcitrant to automation.

Golden Gate assembly (Engler et al. 2009) was invented in part to circumvent gel

purifications, though not without some cost. Type IIS restriction endonucleases recognize asymmetric sequences but cut outside of them. BsaI, for example, recognizes the sequence GGTCTC and introduces staggered cuts in both strands downstream regardless of sequence, creating 5' overhangs that are four nucleotides long. This capacity to create up to 256 different sticky ends with a single enzyme enables concurrent restriction digests and ligations in a single pot. Such simultaneous reactions will hereafter be called "continuous" to distinguish them from "discontinuous" sequential digestions and ligations. Unlike BioBrick assembly, the Golden Gate method can be used to combine multiple parts in a single reaction. It does not leave the characteristic XbaI/SpeI scar of BioBrick assembly so it is better suited for the fusion of open reading frames.

Golden Gate assembly is not, however, without drawbacks. Any BioBrick part can be adjoined to any other part using standard protocols, including those described here. In contrast, the sticky ends produced by BsaI and other Type IIS restriction enzymes are only compatible with others designed to be complementary. Cloning standards for Type IIS restriction endonucleases, such as MoClo (Weber et al. 2011), Phytobricks (Patron et al. 2015), Golden Braid (Sarrion-Perdigones et al. 2011) or Loop assembly (Pollak et al. 2019), facilitate some repurposing of parts for other devices. The MoClo standard, for example, employs nearly three dozen intermediate vectors, each with a unique pair of restriction sites and overhangs, each 
91 dedicated to a separate category of parts (e.g. promoters, 5' upstream untranslated regions, open

92 reading frames, terminators etc.) (Weber et al. 2011). The BioBrick standard employs a single

93 type of vector (Knight 2003) and a single overhang, created by Type II restriction enzymes XbaI

94 or SpeI, to connect parts. BioBrick assembly experiments are thus relatively easy to plan.

I value the simplicity and universal part compatibility of BioBricks, so I invented a less labor intensive and automation-friendly way to assemble them. The concept that underlies my approach is straightforward and easy to implement. In nature every restriction endonuclease is paired with a corresponding site specific DNA modifying enzyme, most often a methyltransferase (Loenen \& Raleigh 2014). Previous reports have described the use of methyltransferases (Lin \& O'Callaghan 2018) or methylated primers (Chen et al. 2013) to enable Golden Gate assemblies that would otherwise have been impossible. The 2ab assembly method is most relevant to the current study. It utilizes in vivo plasmid methylation and recombination of selectable markers to effect one pot, discontinuous ligations of BglBrick parts using Type II restriction enzymes BgIII and BamHI (Leguia et al. 2013). It is efficient, requires little labor and amenable to automation. Unfortunately, the BglBrick and BioBrick standards are incompatible. Moreover $2 \mathrm{ab}$ assembly requires specialized plasmids encoding pairs of selectable markers. It is nevertheless an important precedent for easier ways to combine BioBrick parts, preferably in existing plasmids.

112 coli strains. Cells co-transformed with BioBrick-compatible plasmids thus add methyl groups to 113 DNA at specific sites (Figure 2). The methylated plasmids are prepared and double digested in 
114 accordance with traditional cloning protocols, except that smaller quantities of DNA are

115 required. The restriction fragments are not gel purified but rather combined and reacted with T4

116 DNA ligase. The undesired ligation products, including the original parental plasmids, are

117 subsequently cut by another pair of restriction enzymes. The desired ligation product (insert-

118 recipient plasmid) is protected from both restriction enzymes, so it alone retains the capacity to

119 transform E. coli.

120

121 Materials and Methods

122 Materials

123

The synthetic methylase genes used in this study (M.EcoRI, M.XbaI, M.Ocy1ORF8430P,

M.PstI, and M.AvaIII) were purchased from IDT (Coralville, IA) as gBlocks. Seakem LE

125 agarose was from Lonza Rockland (Rockland, ME) using lambda HindIII, 100 bp (New England

BioLabs, Ipswich, MA) and 10 bp (Thermo Fisher) ladders as molecular size markers.

127 Restriction enzymes, T4 DNA ligase and pure bacteriophage lambda DNA were from NEB

128 (Ipswich, MA). TempliPhi rolling circle amplification kits were from Cytiva (Marlborough,

MA). MinElute PCR purification and GeneRead Size Selection kits were from Qiagen (Valencia,

CA), as was the QIAcube and the custom protocol (vide infra). E. coli OmniMax2 cells were

131 from Invitrogen. Ethylenediaminetetraacetic acid (EDTA), L-arabinose and L-rhamnose were

132 from Sigma Chemicals (St. Louis, MO); isopropyl $\beta$-D-1-thiogalactopyranoside (IPTG) was

133 from Gold Biotechnology (St. Louis, MO). LB broth (Miller) was from EMD Millipore

134 (Billerica, MA) and Bacto-agar was from BD Difco (Franklin Lakes, NJ).

135

136

Methods

PeerJ reviewing PDF | (2020:04:48149:1:0:NEW 7 Jul 2020) 
137 Subcloning via gel purification

138 Two plasmids were purified in triplicate (from cultures seeded with different colonies)

139 via the Qiagen QIAprep spin miniprep kit. Recipient tagRFP-pUC $(1 \mu \mathrm{g})$ was digested in 1x

140 NEB CutSmart buffer ( $80 \mu \mathrm{L}$ total reaction volume) by EcoRI-HF and XbaI (20 units each), thus

141 releasing a short 15 base pair stuffer fragment ("snippet”); lacI-Ptac-lacO-pUC was similarly

142 digested with EcoRI-HF and SpeI-HF in the same buffer, thereby releasing the lacI-Ptac-lacO

143 insert and pUC donor plasmid. All restriction digests in this study were incubated overnight at

$14437^{\circ} \mathrm{C}$ unless otherwise stated. The desired fragments were separated from the undesired ones in

$1450.8 \%$ LE agarose gels; the bands corresponding to the recipient plasmid tagRFP-pUC and insert

146 tagRFP were excised with a razor blade. The desired DNA was purified from the agarose slices

147 via the QiaQuick gel extraction protocol. The fragments $(20 \mathrm{fmol} \sim 50 \mathrm{ng}$ tagRFP-pUC or $25 \mathrm{ng}$

148 lacI-Ptac-lacO), alone or in combination, were reacted to T4 DNA ligase (3 Weiss units) in 1x

149 NEB buffer containing $1 \mathrm{mM}$ ATP $(20 \mu \mathrm{L}$ total reaction volume $)$ overnight in temperature

150 cycled reactions $\left(30^{\circ} \mathrm{C}\right.$ x $30 \mathrm{sec}, 10^{\circ} \mathrm{C}$ x $30 \mathrm{sec}$ ) (Lund et al. 1996). The ligase was heat killed

151 (10 $\mathrm{min}$ at $\left.65^{\circ} \mathrm{C}\right)$, and the reactions $(1 \mathrm{ng})$ were used to transform chemically competent

152 OmniMax 2 cells $(20 \mu \mathrm{L})$. All experiments employed the same batch of cells made competent by

153 the classical method of Inoue et al. (Inoue et al. 1990). Transformation efficiency was $3 \mathrm{x}$

$15410^{7} / \mu \mathrm{g}$, as determined by counting colonies after transformation with $10 \mathrm{pg}$ of pUC19.

155

156 Tip Snip subcloning

157

The lacI-Ptac-lacO-pUC donor plasmid $(1 \mu \mathrm{g})$ in $1 \mathrm{x}$ NEB CutSmart buffer $(80 \mu \mathrm{L}$ total

reaction volume) was shortened slightly by an extra restriction enzyme (20 units PstI-HF) that

159 recognizes a site adjacent to those used to release the insert (20 units each of EcoRI-HF and 
160 SpeI-HF) (Matsumura 2017). The tagRFP-pUC recipient plasmid (1 $\mu \mathrm{g})$ was cut as usual (20

161 units each of EcoRI-HF and XbaI in 1x NEB CutSmart buffer, $80 \mu \mathrm{L}$ total reaction volume). The

162 small restriction fragments ("snippets") in both digests are denatured, annealed to exogenously

163 added anti-snippet oligonucleotides (100 nM BioBrick suffix in the donor digestion, $100 \mathrm{nM}$

164 BioBrick prefix in the recipient digestion), thereby inactivating their sticky ends, and eliminated

165 via Qiagen GeneRead size selection silica spin column chromatography. The purified restriction

166 fragments were ligated (20 fmol 60 ng tagRFP-pUC, 90 ng lacI-Ptac-lacO + pUC, 50 nM PstI

167 "unlinker") in temperature cycled NEB T4 DNA ligase buffer (20 $\mu$ L total reaction volume)

168 prior to heat killing and transformation of E. coli as described above.

$170 \quad 3 \mathrm{~A}$ assembly

A BioBrick-compatible plasmid that encodes chloramphenicol acetyltransferase, RP4 oriT-pUC57-mini-cat $(2 \mu \mathrm{g})$ in $1 \mathrm{x}$ NEB CutSmart buffer ( $80 \mu \mathrm{L}$ total reaction volume) by 20 units each of EcoRI-HF, PstI-HF and NotI-HF (so as to eliminate the sticky ends of its stuffer

174 fragment), dephosphorylated in reactions with NEB Calf Intestinal Phosphatase. The lacI-Ptac-

175 lacO-pUC donor plasmid (300 ng) in 1x NEB 2.1 buffer (15 $\mu \mathrm{L}$ total reaction volume) was

176 digested with 6 units each of EcoRI-HF and SpeI; the tagRFP-pUC donor plasmid was similarly

177 digested with $\mathrm{XbaI}$ and PstI. The digests containing pUC-mini-cat recipient vector (60 ng), the

178 lacI-Ptac-lacO and tagRFP-pUC inserts (50 ng each) were reacted in a thermocycler with 3

179 Weiss units of T4 DNA ligase in 1x NEB T4 DNA ligase buffer (10 $\mu$ L total reaction volume). 180

181 Construction of DNA methyltransferase expression vectors 

aadA) were constructed as follows. BioBrick compatible DNA methyltransferase genes were synthesized without internal BioBrick restriction sites (EcoRI, NotI, XbaI, SpeI or PstI), cloned plasmid origin and spectinomycin resistance marker (aadA) were subcloned from pACYC Duet and pCDF Duet (EMD Millipore, Novagen) respectively into a BioBrick compatible plasmid. The intergenic region between rhaS and rhaB, which includes promoters and operators for both genes, was previously described (Matsumura 2017).

The p15A, aadA, Prham and methylase genes were assembled by a combination of from BioBricks containing these parts prevented efficient digests of the plasmids with XbaI or SpeI-HF. Those plasmids were amplified in vitro by utilizing the TempliPhi rolling circle protocol. The resulting unmethylated amplification product was subsequently digested, and the desired part was gel purified and ligated to other parts. The BioBrick restriction enzymes (EcoRI, XbaI, SpeI and PstI) were eliminated by digesting the plasmids (or amplified versions of them) with XbaI and SpeI-HF, self-ligating the p15A-aadA-Prahm-methylase and using ligation reaction products to transform E. coli OmniMax2. All five methylase expression vectors, plus Prham-tagRFP-pUC, which was used to optimize the optimal concentration of glucose for autoinduction, have been deposited in the Addgene repository (RRID:Addgene_149338 - 149343). 
reacted with XbaI, SpeI (6 units each) and T4 DNA ligase (3 Weiss units) in 1x NEB CutSmart

207

208

209

210

211

212

213

214

215

216

217

218

219

220

221

222

223

224

225

226

227

buffer supplemented with $1 \mathrm{mM}$ ATP $(25 \mu \mathrm{L}$ total reaction volume) in a single pot reaction analogous to that of Golden Gate assembly $\left(72\right.$ cycles of 5 min. at $37^{\circ} \mathrm{C}$, followed by a nested 10 cycles of $30 \mathrm{sec}$ at $10^{\circ} \mathrm{C}$ and $30 \mathrm{sec}$ at $30^{\circ} \mathrm{C}$ ). The reaction was incubated for another hour at $37^{\circ} \mathrm{C}$, then heat killed for $10 \mathrm{~min}$ at $65^{\circ} \mathrm{C} ; 1 \mathrm{ng}$ of total DNA was used to transform $20 \mu \mathrm{L}$ competent E. coli OmniMax 2 cells.

4R/2M (PstI)

M.EcoRI-protected lacI-Ptac-lacO-pUC (500 ng) was digested overnight at $37^{\circ} \mathrm{C}$ by 6 units of SpeI and 8 units of PstI in 1x NEB 2.1 buffer ( $25 \mu \mathrm{L}$ total reaction volume). M.Ocy1protected tagRFP-pUC was similarly digested by 8 units of XbaI and 12 units of PstI. Note that PstI-HF cannot be heat-killed, nor is SpeI-HF fully active in NEB 2.1 buffer, so PstI and SpeI were utilized instead. The restriction enzymes were heat-killed $\left(20 \mathrm{~min}\right.$ at $\left.80^{\circ} \mathrm{C}\right)$, and the restriction fragments (45 ng tagRFP-pUC, 10 ng tagRFP + pUC) were reacted to T4 DNA ligase (2.4 Weiss units in 1x NEB 2.1 buffer supplemented with $1 \mathrm{mM}$ ATP, $20 \mu \mathrm{L}$ total reaction volume) overnight in a thermocycler $\left(600\right.$ cycles of $30 \mathrm{sec}$ at $30^{\circ} \mathrm{C}, 30 \mathrm{sec}$ at $\left.10^{\circ} \mathrm{C}\right)$. The ligase was heat killed by incubation at $65^{\circ} \mathrm{C}$ for $10 \mathrm{~min}$. A $2 \mu \mathrm{L}$ aliquot of each ligation was diluted into a $26 \mu \mathrm{L}$ 1x NEB 2.1 buffer containing 8 units each of EcoRI-HF and SpeI. The post-ligation digest was incubated for 3 hours at $37^{\circ} \mathrm{C}$, and $1 \mu \mathrm{L}$ of the reaction was used to transform $20 \mu \mathrm{L}$ of competent E. coli OmniMax 2 cells.

\section{Results}


228 Subcloning via gel purification as a gold standard

229 Established subcloning methods (Matsumura 2015) were initially applied to set

230 quantitative benchmarks for efficiency (number of correctly assembled clones per ng ligated

231 DNA) and accuracy (fraction of correctly assembled clones among total). Efficiency is important

232 because it is an indirect measure of reliability when optimal conditions cannot be achieved. Two

233 plasmids, lacI-Ptac-lacO-IMBB2.4-pUC57-mini and tagRFP-IMBB2.4-pUC57-mini (hereafter

234 abbreviated lacI-Ptac-lacO-pUC and tagRFP-pUC respectively) were selected as models for this

235 study (Figure 3). Both comply with requirements for established BioBrick RFC[10] assembly

236 protocols. Colonies of cells transformed with the desired assembly product, lacI-Ptac-lacO-

237 tagRFP-pUC, turn pink due to leaky expression of the fluorescent marker protein. Throughout

238 this study, the same E. coli strains, DNA purification techniques, restriction enzymes, ligases and

239 reaction buffers were used, generally in accordance with manufacturer's instructions except as

240 noted. Differences in outcome can thus be attributed solely to differences in assembly protocols.

241 Each cloning step was carried out in triplicate, starting with individual isolated bacterial

242 colonies; standard errors are reported as a measure of variation between experimental replicates.

The most labor-intensive steps of a traditional subcloning experiment are the separation

of restriction fragments via agarose gel electrophoresis, excision of bands corresponding the desired fragments and the extraction of DNA from the agarose slice. Overnight incubations of

247 transformed bacteria, restriction digests and temperature cycled ligation reactions were rate-

248 limiting. The aim here was not to accelerate the workflow, but rather to decrease labor input and

249 increase throughput without compromising efficiency or accuracy. After restriction digests, gel

250 purification and ligation, transformation of $E$. coli with the ligation products led to the growth of 
$251126 \pm 44$ pink colonies per ng; a minority of white colonies $(11 \pm 4=8 \%)$ grew on those LB-

252 ampicillin plates (Table 1). The background on control plates spread with cells transformed with

253 vector only ligations was low ( $\pm 2 \mathrm{cfu} / \mathrm{ng}$ ), which suggested that restriction digests were nearly

254 complete. The insert only ligation controls produced greater background ( $61 \pm 27 \mathrm{cfu} / \mathrm{ng})$, which

255 suggests that the insert was not effectively separated from the donor plasmid in this experiment.

256 Two other established subcloning techniques, tip snip (Matsumura 2017) and 3A (Shetty et al.

257 2011), were also used to provide standards of comparison (Supplemental Material, Table 1).

Methylase expression vectors

The overarching strategy of this study is to replace the gel purification step of subcloning by a combination of site-specific DNA methylation and post-ligation restriction digestion (Spear 2000; Zeng et al. 1997). To realize this strategy, BioBrick compliant genes encoding the DNA methyltransferases of the EcoRI, XbaI and PstI restriction modification systems were synthesized, cloned into compatible plasmids and sequenced. The complete sequence of SpeI methylase (M.SpeI) is not available on REbase (Roberts et al. 2010), so a putative ortholog

M.Ocy1ORF8430P (hereafter abbreviated M.Ocy1) was synthesized instead. Each DNA

et al. 1987), tac (de Boer et al. 1983) and rhamnose operon (Egan \& Schleif 1993) promoters and a strong ribosome binding site. consisting only of the $\mathrm{p} 15 \mathrm{~A}$ replication origin, which is low in copy number and compatible with more common plasmids that encode the pUC origin, and streptomycin 3"-adenylyltransferase 
274 (aadA) selectable marker (Figure 3). The new expression plasmids (promoter-methylase-p15A-

275 aadA) confer resistance to streptomycin and spectinomycin. They don't contain any of the

276 restriction sites normally used for BioBrick assembly (e.g. EcoRI, XbaI, SpeI or PstI) so they

277 won't release any restriction fragments that would interfere with any downstream subcloning

278 steps.

279

280

The in vivo methylase activities produced by these expression vectors was tested as

281 follows. E. coli strain OmniMax 2 was co-transformed with each vector and another BioBrick

282 compatible plasmid, propagated to mid-log culture and induced (either with IPTG or L-

283

284

285

286

287

288

289

290

291

292

293

294

295

296

rhamnose) for three hours. The plasmids were purified and reacted with restriction

endonucleases including the one normally associated with each DNA methyltransferase in wild-

type bacteria. The degree of protection was assessed by comparing the mobilities in agarose gels

of plasmids that were uncut, completely cut by a restriction endonuclease unrelated to the

methylase or protected at least in part by in vivo methylation. For example, agarose gel

electrophoresis showed that lacI-Ptac-lacO-pUC purified from E. coli carrying Prham-M.XbaI-

p15A-aadA was digested by SpeI but mostly resistant to XbaI. Conversely, tagRFP-pUC

protected by Prham-M.Ocy1-p15A-aadA was digested with XbaI but mostly resistant to SpeI

(Figure 4).

The rhamnose promoter, reputedly the weakest of the three tested, proved most reliable

for consistent and complete in vivo methylation. I speculate that DNA methyltransferases that are site-specific at moderate concentrations become toxic to host cells when over-expressed (Bandaru et al. 1996). Extended over-expression could thus favor the accumulation of mutations 
297 beneficial to transformed cells but unwanted by human scientists. Induction of transformants at 298 mid-log phase is itself labor-intensive, as cultures propagated in parallel don't always grow at the

299 same rate, so an auto-induction protocol was developed. The rhamnose promoter is regulated by

300 catabolite repression as well as by L-rhamnose. The plasmid Prham-tagRFP-pUC (Matsumura

301 2017) was used to transform E. coli OmniMax 2. Limiting amounts of glucose were added to

302 saturating concentrations of L-rhamnose (0.1\%) in LB medium supplemented with ampicillin.

303 Commercial LB contains varying quantities of glucose, but for the addition of $0.001 \%$ glucose to

$3040.1 \%$ L-rhamnose led to maximum tagRFP expression as measured in a microtiter plate

305 spectrofluorimeter. Autoinduction under those growth conditions led complete in vivo

306 methylation when the methylase expression vectors were used instead.

307

308

The other lesson inferred from the in vivo methylation experiments was that M.PstI is rarely able to methylate plasmids within E. coli cells as completely as M.EcoRI, M.XbaI or M.Ocy1. Each of these methylases evolved in a different bacterial species so it isn't surprising that one of the four proved less active than the others in the alien environment of the $E$. coli cytoplasm. Most of our plasmids include an NsiI site adjacent to the PstI site. The sequence of M.NsiI was not available on REbase (Roberts et al. 2010) so the gene encoding the M.AvaIII ortholog was synthesized, cloned, sequenced and subcloned downstream of the rhamnose than did M.PstI.

317

318

2RM assembly 

assembly experiments. The 3A BioBrick assembly protocol (Shetty et al. 2011) was so named

321 because it employs three plasmids, each with a distinct antibiotic selection marker. For similar

322 reasons, 2RM assembly utilizes the components of two restriction modification systems:

323

324

restriction endonucleases $\mathrm{XbaI}$ and SpeI-HF, and DNA methyltransferases M.XbaI and M.SpeI homologue M.Ocy1 (Figure 5). In this embodiment, the lacI-Ptac-lacO-pUC was purified from triplicate cultures of auto-induced cells containing Prham-M.XbaI-p15A-aadA, while tagRFPpUC was purified from cultures co-transformed with Prham-M.Ocy1-p15A-aadA. The purified plasmids were mixed and reacted with $\mathrm{XbaI}$ and SpeI. Each plasmid, lacI-Ptac-lacO-pUC and tagRFP-pUC, was cut with one of the two restriction enzymes and protected by methylation from the other. The linearized plasmids (Figures 5 and S1) react with T4 DNA ligase to form three sets of products. Most common, presumably, are the two original parental plasmids. Each of the linearized plasmids can also be ligated to other copies of themselves in one of two orientations to form homodimers (Figures 5 and S2). All contain unmethylated XbaI or SpeI sites, so they are susceptible to re-digestion by the restriction enymes in the reaction vessel. The linearized plasmids can also ligation to each other to form heterodimers (Figures 5 and S3). These products are resistant to both restriction endonucleases so they should accumulate over the course of the digestion/ligation reaction.

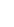

When E. coli were transformed with one nanogram of each ligation reaction, $118 \pm 13$ pink cfu/ng and $260 \pm 25$ white cfu/ng were observed on each plate (Table 1). Colony numbers on plates corresponding to control ligations with only one plasmid (20 $\pm 4 \mathrm{cfu} / \mathrm{ng})$ or the other (4 $\pm 1 \mathrm{cfu} / \mathrm{ng}$ ) were relatively low, suggesting that both methylation and restriction digestion was 
342 nearly complete. These results in combination show that restriction digestion of the parental

343 plasmids and homodimeric ligation products was efficient, and that ligation to form

344 heterodimeric products was also efficient. In principle, the ratio of pink to white colonies should

345 be 1:1, but the 1:2.2 ratio observed here could mean that the ligation product with the undesired

346 orientation conferred greater fitness upon the host cell. The desired product contains two copies

347 of the selectable marker and origin of replication (Figures 5 and S3), which could complicate

348 subsequent assembly reactions. Double digests of existing BioBrick-compatible plasmids enable

349 directional cloning, which is more practical.

350

351

4R/2M (PstI) assembly

352

In 4R/2M assembly, the two parental plasmids are sequentially reacted with two DNA

353

354

355

356

357

358

359

360

361

362

363

364

methyltransferases, three restriction endonucleases, T4 DNA ligase and a fourth restriction

enzyme (Figure 6). In its 4R/2M (PstI) embodiment, the recipient encodes the part that will end up on the 5' end of the desired ligation product. Its EcoRI site is methylated in vivo and

subsequently digested by SpeI and PstI (Figures 6 and S4). The donor plasmid that encodes the insert destined for the 3' end of the desired ligation product; it is protected from SpeI by M.Ocy1 and separately double digested by $\mathrm{XbaI}$ and PstI. The restriction endonucleases in both digestion reactions are subsequently heat-killed $\left(20 \mathrm{~min}\right.$. at $\left.80^{\circ} \mathrm{C}\right)$; the four digestion products are combined and reacted with T4 DNA ligase and ATP. The ligase is then heat-killed, and the ligation products (Figures 6, S5 and S6) are diluted and further digested with EcoRI and SpeI.

EcoRI linearizes the undesired donor plasmid and any ligation product that includes it. SpeI linearizes the other parental plasmid, so that the desired insert-recipient plasmid ligation 
365 product is the only viable construct that remains intact. Homodimeric constructs are produced in

366 any ligation of fragments produced by type II restriction endonucleases (Figure 1), but none are

367 viable in vivo because plasmids are destabilized by large inverted repeats. Competent E. coli

368 were transformed with the $4 \mathrm{R} / 2 \mathrm{M}$ (PstI) assembly reactions, leading to the formation $177 \pm 4$

$369 \mathrm{pink} \mathrm{cfu} / \mathrm{ng}$ and only $2 \pm 1$ white cfu/ng (Table 1 ). Background colony counts on the control

370 plates representing vector only $(1 \pm 0.2 \mathrm{cfu} / \mathrm{ng})$ and insert only $(1 \pm 0.2 \mathrm{cfu} / \mathrm{ng})$ ligations were

371 very low. The 4R/2M (PstI) assembly is thus well suited for routine high throughput BioBrick

372 assembly. I have subsequently used it to assemble 65 more pairs of BioBricks in batches of up to 37318.

4R/2M (EcoRI) assembly

The logic of 4R/2M (EcoRI) BioBrick assembly is identical to that of 4R/2M (PstI), except that the recipient and donor plasmids are switched. The BioBrick part that ends up on the $5^{\prime}$ end of the assembled product is the insert rather than part of the recipient plasmid. The recipient tagRFP-pUC was methylated in vivo by M.PstI; 600 ng was double digested by EcoRI$\mathrm{HF}$ and XbaI (12 units each in $30 \mu \mathrm{L}$ NEB CutSmart buffer). Donor lacI-Ptac-lacO-pUC was protected by M.XbaI prior to purification; 600 ng was similarly digested with EcoRI-HF and SpeI-HF (Figure S7). The restriction enzymes in both digests were heat-killed $\left(20 \mathrm{~min}\right.$. at $\left.80^{\circ} \mathrm{C}\right)$ and the restriction fragments (50 ng tagRFP-pUC, 90 ng lacI-Ptac-lacO) were mixed and reacted overnight in a thermocycler with T4 DNA ligase (3 Weiss units in $25 \mu \mathrm{L}$ NEB CutSmart buffer supplemented with $1 \mathrm{mM}$ ATP). The enzyme was heat-killed $\left(10 \mathrm{~min}\right.$. at $\left.65^{\circ} \mathrm{C}\right)$, and the ligation product $(1 \mathrm{ng} / \mu \mathrm{L})$ digested with 8 units each PstI-HF and XbaI in NEB CutSmart buffer (Figures S8 and S9). The transformation of competent E. coli cells produced only $19 \pm 7$ pink colonies, 
388 significantly less than the $4 \mathrm{R} / 2 \mathrm{M}$ (PstI) experiment with the same plasmids, and $8 \pm 6$ white 389 colonies per ng (Table 1). As previously noted, M.PstI does not methylate in vivo as reliably as 390 our other DNA methyltransferases.

391

392

393

394

395

396

397

398

399

400

401

402

403

404

405

406

407

408

409

410

The assembly was repeated, except that the tagRFP-pUC plasmid was reacted in vivo

with M.AvaIII instead of M.PstI. M.AvaIII catalyzes the methylation of NsiI sites, which exist in most BioBrick compatible plasmids in my lab (Matsumura 2017). NsiI produces sticky ends compatible with those of PstI so it offers a good comparison. This assembly, after digestion with NsiI and XbaI, produced $299 \pm 91$ pink colonies and only $12 \pm 3$ white colonies per ng (Table 1). This improved result in consistent with the hypothesis that $4 \mathrm{R} / 2 \mathrm{M}$ assembly can be limited by the degree to which the populations of plasmids purified from E. coli are methylated.

Discussion

The assembly protocols described here could be further improved in several ways. The $4 \mathrm{R} / 2 \mathrm{M}(\mathrm{EcoRI})$ is more efficient when M.AvaIII expression vectors were employed instead of those that produce M.PstI. Not all BioBrick compatible plasmids contain NsiI sites, so in vivo M.PstI activity could be enhanced, either by optimizing expression via directed evolution (using in vitro PstI activity as a selection), co-expression with the PstI restriction endonuclease (as in the wild-type operon) or by identifying an M.PstI ortholog that is more active in the E. coli cytoplasm. Another alternative is to clone and express another site-specific DNA methyltransferase that protects some other site that is common in plasmid backbones but very rare within inserts. The tactic of using pairs of methylases to protect desired insert-recipient plasmids from double digests following ligation need not be restricted to BioBrick assembly. It 
411 could potentially be generalized to streamline other kinds of subcloning experiments if the

412 relevant DNA methyltransferase expression vectors were available.

413

414

The $2 \mathrm{RM}$ assembly method is a single pot continuous reaction for the restriction more of the recombinant plasmids are ligated in the undesired orientation. The utility of the

existing protocol is limited, but it offers some evidence that continuous assembly of correctly oriented ligation products is possible. Such a process would probably require a more elaborate variant of the BioBrick standard and plasmids methylated at more than one restriction site. If

four Type II restriction endonucleases and T4 DNA ligase work together efficiently, two mixing steps (heat killing restriction enzymes, ligation reaction setup) of the $4 \mathrm{R} / 2 \mathrm{M}$ protocol would be obviated. This hypothetical assembly process would retain the simplicity of the BioBrick standard but emulate the ease of use of Golden Gate.

Conclusions

The 4R/2M (PstI) BioBrick assembly described above is less labor-intensive and easier to scale up than is the traditional gel purification approach. It is more efficient and accurate than is 3A assembly and requires less reagents than does Tip Snip subcloning. The value of the labor savings is proportional to the number of assemblies that can be conducted in parallel. The $4 \mathrm{R} / 2 \mathrm{M}$ procedure was not designed to match the convenience of single pot, continuous Golden Gate assembly, but BioBrick assembly experiments are arguably easier to design and debug. The BioBrick standard thus remains well suited for the high school and undergraduate students who participate in iGEM competitions. The throughput of $4 \mathrm{R} / 2 \mathrm{M}$ BioBrick assembly is mostly 
434 limited by the numbers of plasmid minipreps that users can perform in parallel. The quantity of

435 plasmid required is relatively low $(\leq 400 \mathrm{ng} /$ digest, as opposed to $1-2 \mu \mathrm{g}$ for gel purification or

436 Tip Snip) because none is lost during subsequent spin column chromatography. This

437 methodological advance should thus accelerate the work of the BioBricks user community and 438 encourage others to join.

\section{References}

441

442

Bandaru B, Gopal J, and Bhagwat AS. 1996. Overproduction of DNA cytosine methyltransferases causes methylation and C --> T mutations at non-canonical sites. $J$ Biol Chem 271:7851-7859. 10.1074/jbc.271.13.7851

Bryksin AV, and Matsumura I. 2010. Overlap extension PCR cloning: a simple and reliable way to create recombinant plasmids. Biotechniques 48:463-465. 10.2144/000113418

Bujard H, Gentz R, Lanzer M, Stueber D, Mueller M, Ibrahimi I, Haeuptle MT, and Dobberstein B. 1987. A T5 promoter-based transcription-translation system for the analysis of proteins in vitro and in vivo. Methods Enzymol 155:416-433. 10.1016/00766879(87)55028-5

Casini A, Storch M, Baldwin GS, and Ellis T. 2015. Bricks and blueprints: methods and standards for DNA assembly. Nat Rev Mol Cell Biol 16:568-576. 10.1038/nrm4014

Chao R, Yuan Y, and Zhao H. 2015. Recent advances in DNA assembly technologies. FEMS Yeast Res 15:1-9. 10.1111/1567-1364.12171

Chen WH, Qin ZJ, Wang J, and Zhao GP. 2013. The MASTER (methylation-assisted tailorable ends rational) ligation method for seamless DNA assembly. Nucleic Acids Res 41:e93. $10.1093 / \mathrm{nar} / \mathrm{gkt1} 22$ 
457 de Boer HA, Comstock LJ, and Vasser M. 1983. The tac promoter: a functional hybrid derived 458 from the trp and lac promoters. Proc Natl Acad Sci U S A 80:21-25. 10.1073/pnas.80.1.21

459 Egan SM, and Schleif RF. 1993. A regulatory cascade in the induction of rhaBAD. J Mol Biol $460 \quad 234: 87-98.10 .1006 /$ jmbi.1993.1565

461 Engler C, Gruetzner R, Kandzia R, and Marillonnet S. 2009. Golden gate shuffling: a one-pot

462

463

464

465

466

467

468

469

470

471

472

473

474

475

476

477

478

479

DNA shuffling method based on type IIs restriction enzymes. PLoS One 4:e5553. 10.1371/journal.pone.0005553

Inoue H, Nojima H, and Okayama H. 1990. High efficiency transformation of Escherichia coli with plasmids. Gene 96:23-28. 10.1016/0378-1119(90)90336-p

Knight TF. 2003. Idempotent Vector Design for Standard Assembly of Biobricks. MIT Synthetic Biology Working Group.

Kosuri S, and Church GM. 2014. Large-scale de novo DNA synthesis: technologies and applications. Nat Methods 11:499-507. 10.1038/nmeth.2918

Kuhn P, Wagner K, Heil K, Liss M, and Netuschil N. 2017. Next generation gene synthesis: From microarrays to genomes. Eng Life Sci 17:6-13.

Leguia M, Brophy JA, Densmore D, Asante A, and Anderson JC. 2013. 2ab assembly: a methodology for automatable, high-throughput assembly of standard biological parts. $J$ Biol Eng 7:2. 10.1186/1754-1611-7-2

Lin D, and O'Callaghan CA. 2018. MetClo: methylase-assisted hierarchical DNA assembly using a single type IIS restriction enzyme. Nucleic Acids Res 46:e113. 10.1093/nar/gky596

Loenen WA, and Raleigh EA. 2014. The other face of restriction: modification-dependent enzymes. Nucleic Acids Res 42:56-69. 10.1093/nar/gkt747 
480 Lund AH, Duch M, and Pedersen FS. 1996. Increased cloning efficiency by temperature-cycle

481

482

483

484

485

486

487

488

489

490

491

492

493

494

495

496

497

498

499

500

501 ligation. Nucleic Acids Res 24:800-801. 10.1093/nar/24.4.800

Matsumura I. 2015. Why Johnny can't clone: Common pitfalls and not so common solutions. Biotechniques 59:IV-XIII. 10.2144/000114324

Matsumura I. 2017. Semi-automated Tip Snip cloning of restriction fragments into and out of plasmid polylinkers. Biotechniques 62:99-106. 10.2144/000114522

Patron NJ, Orzaez D, Marillonnet S, Warzecha H, Matthewman C, Youles M, Raitskin O, Leveau A, Farre G, Rogers C, Smith A, Hibberd J, Webb AA, Locke J, Schornack S, Ajioka J, Baulcombe DC, Zipfel C, Kamoun S, Jones JD, Kuhn H, Robatzek S, Van Esse HP, Sanders D, Oldroyd G, Martin C, Field R, O'Connor S, Fox S, Wulff B, Miller B, Breakspear A, Radhakrishnan G, Delaux PM, Loque D, Granell A, Tissier A, Shih P, Brutnell TP, Quick WP, Rischer H, Fraser PD, Aharoni A, Raines C, South PF, Ane JM, Hamberger BR, Langdale J, Stougaard J, Bouwmeester H, Udvardi M, Murray JA, Ntoukakis V, Schafer P, Denby K, Edwards KJ, Osbourn A, and Haseloff J. 2015. Standards for plant synthetic biology: a common syntax for exchange of DNA parts. New Phytol 208:13-19. 10.1111/nph.13532

Pollak B, Cerda A, Delmans M, Alamos S, Moyano T, West A, Gutierrez RA, Patron NJ, Federici F, and Haseloff J. 2019. Loop assembly: a simple and open system for recursive fabrication of DNA circuits. New Phytol 222:628-640. 10.1111/nph.15625

Roberts RJ, Vincze T, Posfai J, and Macelis D. 2010. REBASE--a database for DNA restriction and modification: enzymes, genes and genomes. Nucleic Acids Res 38:D234-236. $10.1093 /$ nar/gkp874 
502 Sands B, and Brent R. 2016. Overview of Post Cohen-Boyer Methods for Single Segment 503 Cloning and for Multisegment DNA Assembly. Curr Protoc Mol Biol 113:3 26 21-23 26

504 20. $10.1002 / 0471142727 . \mathrm{mb} 0326 \mathrm{~s} 113$

505 Sarrion-Perdigones A, Falconi EE, Zandalinas SI, Juarez P, Fernandez-del-Carmen A, Granell A, 506 and Orzaez D. 2011. GoldenBraid: an iterative cloning system for standardized assembly of reusable genetic modules. PLoS One 6:e21622. 10.1371/journal.pone.0021622

508

509

510

511

512

513

514

515

516

517

518

519

520

521

522

523

524

Shetty R, Lizarazo M, Rettberg R, and Knight TF. 2011. Assembly of BioBrick standard biological parts using three antibiotic assembly. Methods Enzymol 498:311-326. 10.1016/B978-0-12-385120-8.00013-9

Spear MA. 2000. Efficient DNA subcloning through selective restriction endonuclease digestion. Biotechniques 28:660-662, 664, 666 passim. 10.2144/00284st01

Vazquez-Vilar M, Orzaez D, and Patron N. 2018. DNA assembly standards: Setting the lowlevel programming code for plant biotechnology. Plant Sci 273:33-41. 10.1016/j.plantsci.2018.02.024

Watson JF, and Garcia-Nafria J. 2019. In vivo DNA assembly using common laboratory bacteria: A re-emerging tool to simplify molecular cloning. J Biol Chem 294:1527115281. 10.1074/jbc.REV119.009109

Weber E, Engler C, Gruetzner R, Werner S, and Marillonnet S. 2011. A modular cloning system for standardized assembly of multigene constructs. PLoS One 6:e16765. 10.1371/journal.pone.0016765

Zeng Q, Eidsness MK, and Summers AO. 1997. Near-zero background cloning of PCR products. Biotechniques 23:412-414, 416, 418. 10.2144/97233bm13 


\section{Figure Legends}

\section{Figure 1. Conventional subcloning of BioBrick-compatible parts}

528 (Top) Recipient (blue) and donor (green) plasmids both contain inserts bound by the same

529 restriction sites $(\mathrm{E}=\mathrm{EcoRI}, \mathrm{X}=\mathrm{XbaI}, \mathrm{S}=\mathrm{SpeI}, \mathrm{P}=\mathrm{PstI})$. (Middle row) The recipient plasmid is

530 cut with SpeI and PstI, releasing a short stuffer fragment; the donor is separately cut with XbaI or

531 PstI, so that insert is released from plasmid. The fragments from both digests are separated by

532 agarose gel electrophoresis. The desired plasmid and insert are excised from the gel and

533 subsequently purified; the unwanted stuffer and donor plasmid (both within dashed lined

534 rectangles) are left in the gels and thrown away. (Bottom) The recipient plasmid and insert are

535 ligated together forming three products: (left) the plasmid homodimer, (middle) the insert

536 homodimer and (right) insert-recipient plasmid heterodimer. Large inverted repeats cannot

537 replicate stably so the desired insert-plasmid (bottom right) is the only product capable of

538 conferring antibiotic selection if the digests and ligations were efficient.

539

540

Figure 2. Subcloning of a methylated insert into a methylated recipient plasmid.

541 (Far left) Donor (blue) or recipient (red) plasmids are purified from Escherichia coli strains that

542 express foreign DNA methyltransferases that protect restriction sites C or D, respectively.

543 Modified sites are shown in parentheses. (Middle left) Both plasmids are reacted with restriction

544 enzymes A and B, thereby producing four fragments: (top to bottom) insert (blue, methylated),

545 donor plasmid (blue unmethylated), stuffer (red unmethylated), and recipient plasmid (red

546 methylated). All the restriction fragments are ligated. Two recapitulate the parental plasmids

547 (mixed blue and red at far left). (Middle right) Four are homodimers (fragments ligating to other

548 copies of themselves, all blue or red). The insert homodimer resists further digestion but lacks 
549 any selectable marker or origin of replication. The recipient plasmid homodimer also remains

550 circular, but it is a large inverted repeat, so it is not stable in E. coli. Four others are heterodimers

551 (mixed blue and red). Polymeric concatemers (linear trimers, circular tetramers, etc.) also form at

552 low frequency but are not shown. (Far right) Double digestion of the ligation products with

553 restriction enzymes $\mathrm{C}$ and $\mathrm{D}$ linearizes almost all that are circular, except for the desired double

554 methylated insert-recipient plasmid construct. It alone retains the capacity to transform E. coli 555 efficiently.

556

557 Figure 3. Model plasmids used in this study

558 (Top left) The lacI-Ptac-lacO insert includes a promoter that is somewhat leaky at high copy

559 number. The IMBB2.4-pUC57-mini backbone, hereafter abbreviated pUC, is BioBrick-

560 compatible and also includes an NsiI site downstream of PstI (Matsumura 2017). (Top right)

561 The tagRFP reporter protein can cause colonies to turn visibly pink, but only when the gene

562 encoding it is subcloned downstream of a leaky or constitutive promoter. (Bottom left) RP4 oriT-

563 pUC-cat is a BioBrick compatible plasmid that confers resistance to chloramphenicol instead of

564 ampicillin. RP4 oriT serves as a small stuffer in these experiments. In this study this latter

565 plasmid is used only as a recipient plasmid (destination vector) for 3A assembly. (Bottom right)

566 Five expression vectors for production of recombinant DNA methyltransferases were constructed

567 for this study. The version that expresses M.Ocy1ORF8430P, a putative ortholog of M.SpeI, is

568 shown. The others are similar in design but express M.XbaI, M.EcoRI, M.PstI or M.AvaIII

569 instead. Each plasmid utilizes the low copy number p15A origin (pACYC) and confers resistance

570 to spectinomycin and is thus compatible with pUC plasmids that impart resistance to ampicillin,

571 chloramphenicol or kanamycin. The DNA methyltransferase expression vectors do not contain 
572 any of the restriction sites employed in BioBrick assembly protocols (EcoRI, XbaI, SpeI or PstI),

573 so they will not produce restriction fragments that ligate to those that are desired.

574

575 Figure 4. M.XbaI and M.Ocy1ORF8430P protect plasmids from XbaI and SpeI

576 Model plasmids lacI-Ptac-lacO-pUC and tagRFP-pUC were purified from triplicate cultures of

577 E. coli OmniMax 2 co-transformed with Prham-M.XbaI-p15A-aadA or Prham-

578 M.Ocy1ORF8430P-p15A-aadA (Figure 3) respectively. Each purified enzyme was reacted in

579 vitro with $\mathrm{XbaI}$ or SpeI-HF, and the extent to which each was cut was assessed by agarose gel

580 electrophoresis. Each of the DNA methyltransferases appears to protect co-transformed plasmid

581 from its corresponding restriction endonuclease, and that protection is sequence specific.

582

583 Figure 5. 2RM BioBrick assembly

584 (Top) The SpeI site of one cloned BioBrick part, and the XbaI site of another, are methylated in 585 vivo. (Middle) The methylated plasmids are mixed together with XbaI, SpeI and T4 DNA ligase.

586 Each plasmid is digested by one restriction endonuclease and protected from the other. The

587 linear digestion products are self-ligated to form the parental plasmids (top), to another copy of

588 the same molecule in one of two orientations to form a homodimer product (bottom left) or to a

589 copy of the other plasmid (again in one of two possible orientations) to form a heterodimer

590 (bottom right). The parental plasmids and homodimers are susceptible to re-digestion, so they are

591 depleted over time while the heterodimers accumulate.

592

593 Figure 6. 4R/2M (PstI) BioBrick assembly 
594 (Top) The EcoRI site of the recipient plasmid and SpeI site of the insert are methylated in vivo. 595 (Middle) The recipient plasmid is digested with SpeI and PstI, so that it releases a short 18 bp 596 stuffer (or "snippet", not shown). The other plasmid is separately digested with XbaI and PstI, 597 producing the desired insert and the undesired donor. (Bottom) The restriction enzymes are heat598 killed, the digestion products are mixed and reacted with T4 DNA ligase, forming three sets of 599 ligation products: parental plasmids (top), homodimers (bottom left) and heterodimers (bottom 600 right). The $36 \mathrm{bp}$ snippet homodimer is not shown, nor are trimer, tetramer and other higher 601 order products. The homodimer products are large perfect inverted repeats, which are not 602 expected to replicate efficiently in vivo. Moreover, none of the undesired parental, homodimer or 603 heterodimers are resistant to subsequent digestion with EcoRI and SpeI. Only the desired 604 insert/recipient recombinant plasmid (bottom right) retains its ability to transform E. coli. 
Table $\mathbf{1}$ (on next page)

Table 1. Colony counts (cfu/ng) 
1 Table 1. Colony counts (cfu/ng)

\begin{tabular}{|l|l|l|l|l|}
\hline Assembly protocol & Vector only & Insert only & Vector + insert (red) & Vector + insert (white) \\
\hline Gel purify (EcoRI) & $7 \pm 2$ & $61 \pm 27$ & $126 \pm 44$ & $11 \pm 4$ \\
\hline Tip Snip (EcoRI) & $8 \pm 2$ & $9 \pm 6$ & $384 \pm 61$ & $11 \pm 4$ \\
\hline $3 \mathrm{~A}$ & 0 & 0 & $4 \pm 1$ & $5 \pm 2$ \\
\hline 2RM & $20 \pm 4$ & $4 \pm 1$ & $118 \pm 13$ & $260 \pm 25$ \\
\hline 4R/2M (Pstl) & $1 \pm 0.2$ & $1 \pm 0.2$ & $177 \pm 4$ & $2 \pm 1$ \\
\hline 4R/2M (EcoRI) & $0 \pm 0$ & $2 \pm 1$ & $19 \pm 7$ & $8 \pm 6$ \\
\hline 4R/2M (EcoRI,Nsil) & $1 \pm 1$ & $10 \pm 4$ & $299 \pm 91$ & $12 \pm 3$ \\
\hline
\end{tabular}

2 


\section{Figure 1}

Subcloning of a methylated insert into a methylated recipient plasmid.

(Far left) Donor (blue) or recipient (red) plasmids are purified from Escherichia coli strains that express foreign DNA methyltransferases that protect restriction sites $C$ or $D$, respectively. Modified sites are shown in parentheses. (Middle left) Both plasmids are reacted with restriction enzymes $A$ and $B$, thereby producing four fragments: (top to bottom) insert (blue, methylated), donor plasmid (blue unmethylated), stuffer (red unmethylated), recipient plasmid (red methylated). All the restriction fragments are ligated. Two recapitulate the parental plasmids (mixed blue and red at far left). (Middle right) Four are homodimers (fragments ligating to other copies of themselves, all blue or red). The insert homodimer resists further digestion but lacks any selectable marker or origin of replication. The recipient plasmid homodimer also remains circular, but it is a large inverted repeat, so it is not stable in E. coli. Four others are heterodimers (mixed blue and red). Polymeric concatemers (linear trimers, circular tetramers, etc.) also form at low frequency but are not shown. (Far right) Double digestion of the ligation products with restriction enzymes $C$ and $D$ linearizes almost all that are circular, except for the desired double methylated insert-recipient plasmid construct. It alone retains the capacity to transform E. coli efficiently. 


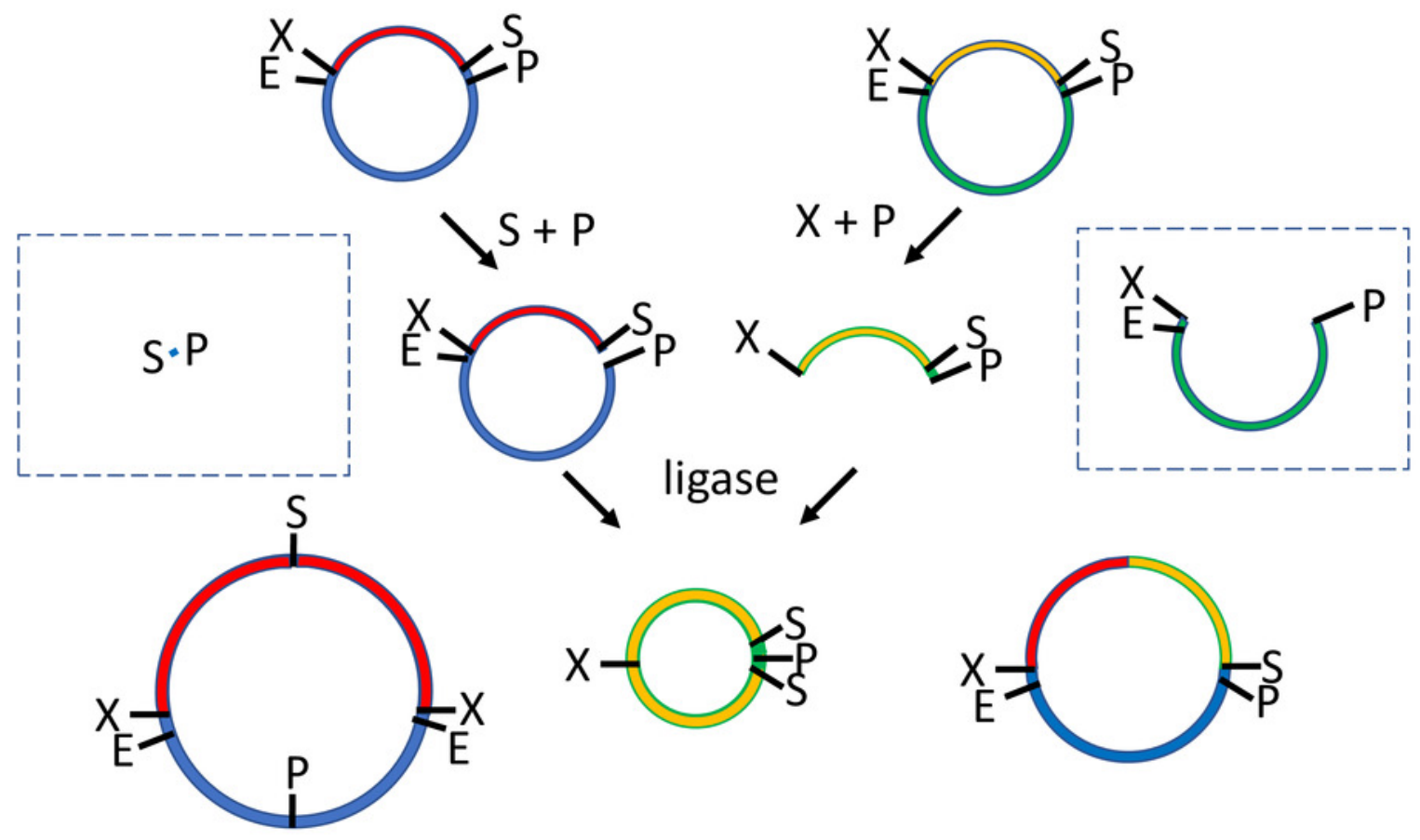




\section{Figure 2}

Model plasmids used in this study

(Top left) The lacl-Ptac-lacO insert includes a promoter that is somewhat leaky at high copy number. The IMBB2.4-pUC57-mini backbone, hereafter abbreviated pUC, is BioBrickcompatible and also includes an Nsil site downstream of Pstl (Matsumura 2017) . (Top right) The tagRFP reporter protein can cause colonies to turn visibly pink, but only when the gene encoding it is subcloned downstream of a leaky or constitutive promoter. (Bottom) RP4 oriTpUC-cat is a BioBrick compatible plasmid that confers resistance to chloramphenicol instead of ampicillin. RP4 oriT serves as a small stuffer in these experiments. In this study this latter plasmid is used only as a recipient plasmid (destination vector) for $3 \mathrm{~A}$ assembly. 


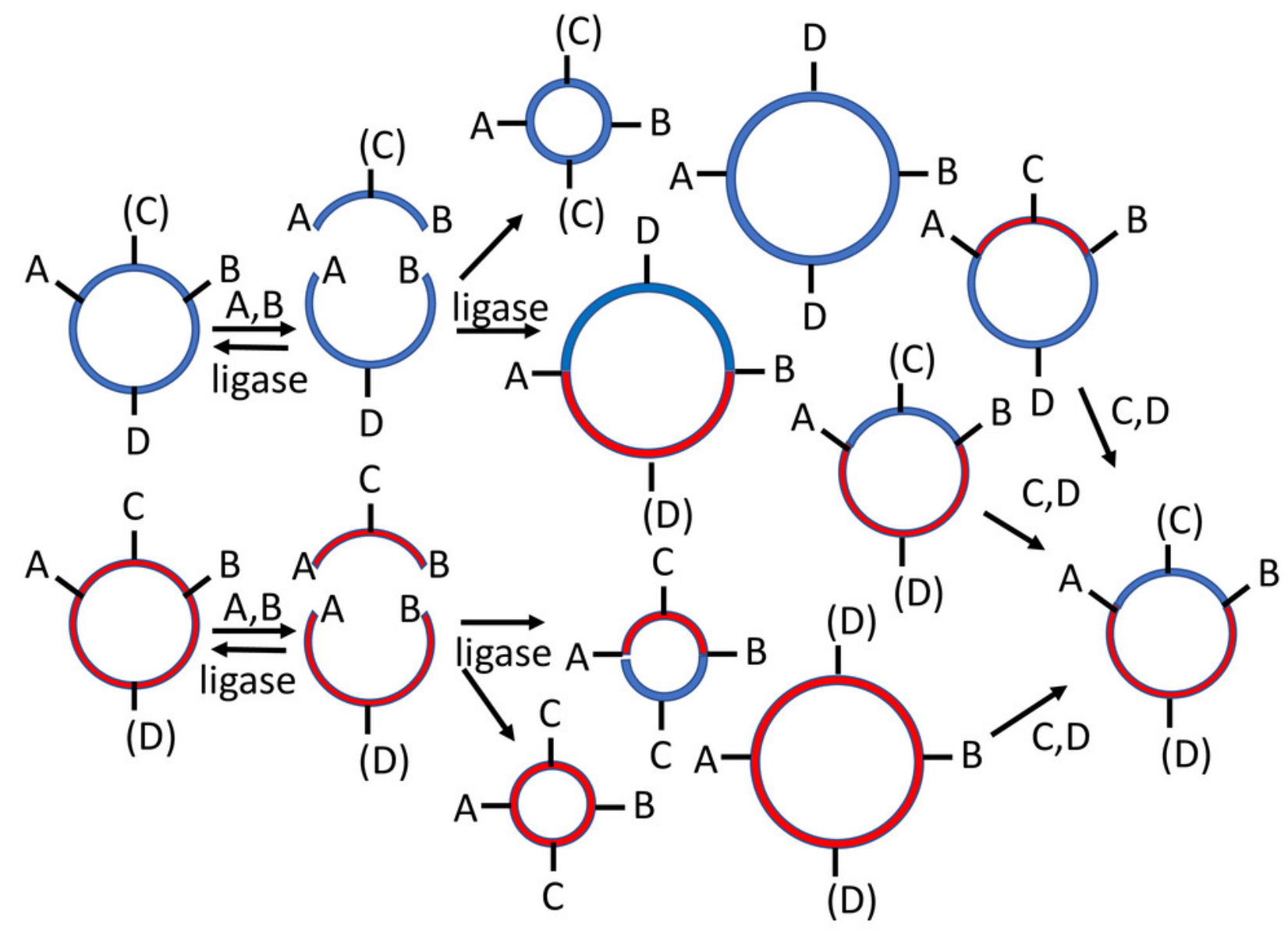


Figure 3

$3 \mathrm{~A}$ restriction fragments

Double digests of three plasmids (Figure 2) produces six restriction fragments, three of which are desired (inserts 1 and 2, recipient plasmid) and three that are not (donor plasmids 1 and 2 , stuffer). Each fragment can ligate to one of four, including another copy of itself (not shown).
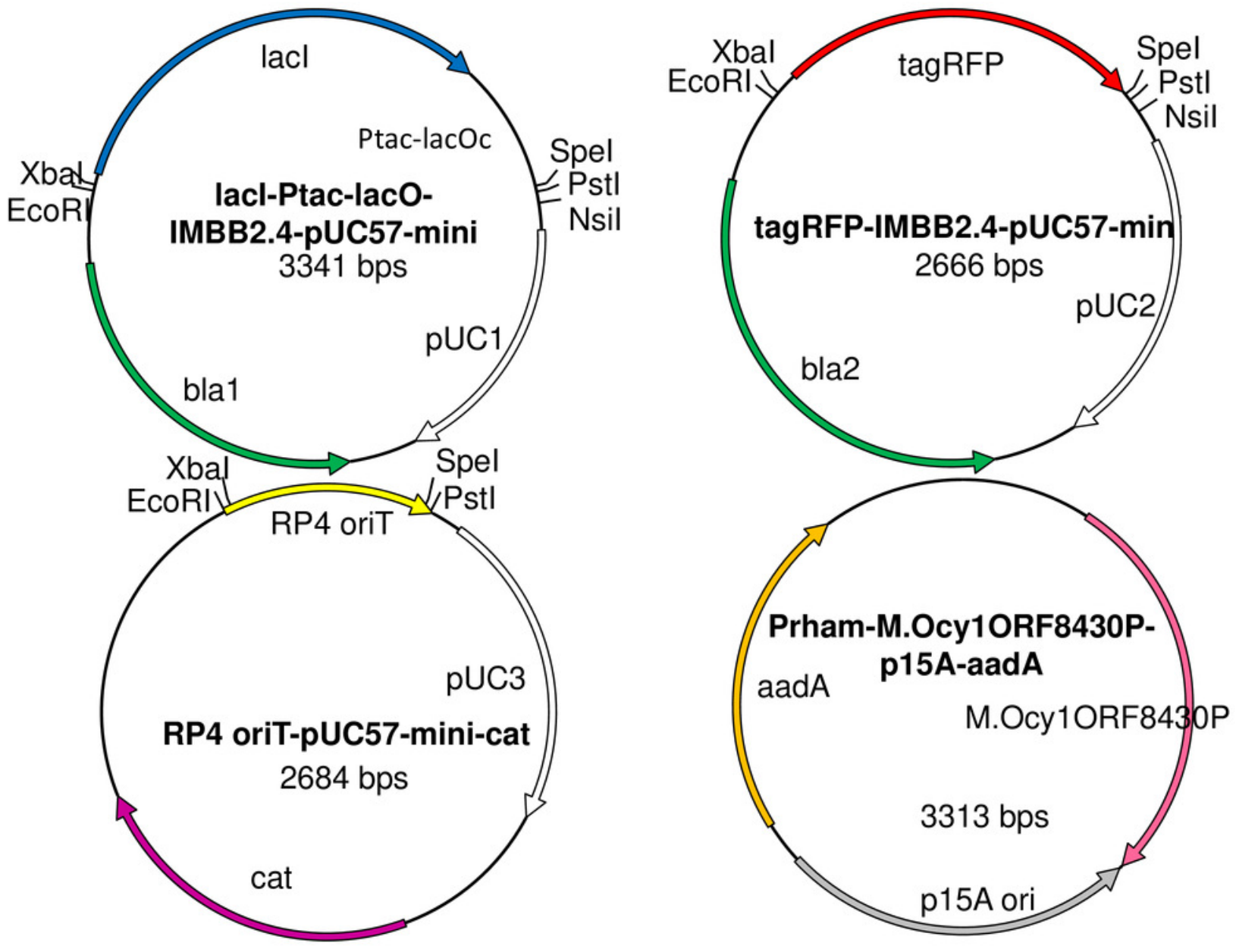
Figure 4

viable trimeric $3 \mathrm{~A}$ ligation products

The six restriction fragments produced during $3 \mathrm{~A}$ assembly can ligate to each other to produce a variety of products (not shown). Only those that are circular and contain the right selectable marker (chloramphenicol acetyltransferase in this case) are viable. Still, most $E$. coli colonies will carry undesired ligation products (top and bottom left) so colony screening is required to identify the desired construct (bottom right). 


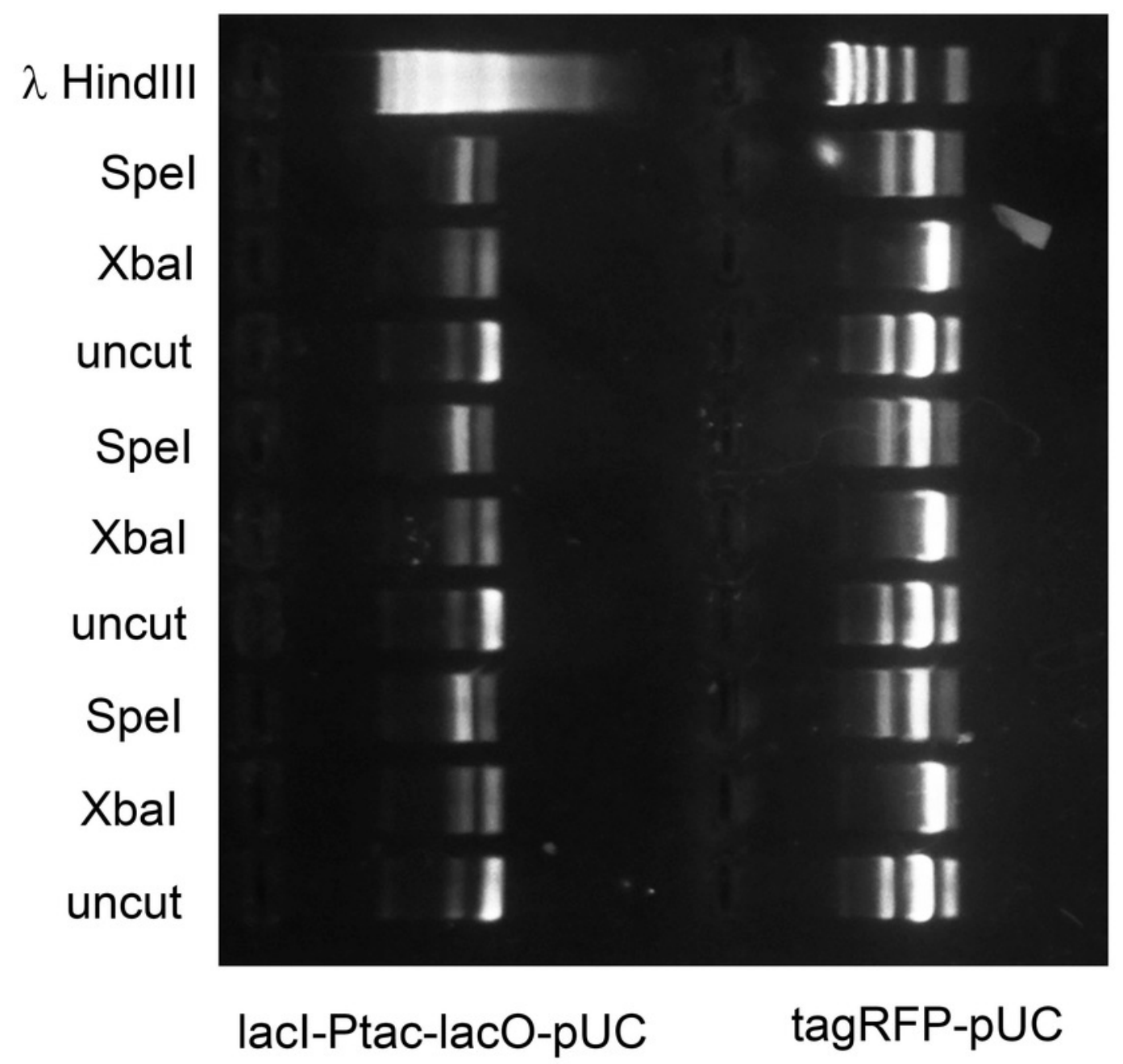




\section{Figure $\mathbf{5}$ (on next page)}

DNA methyltransferase expression vector

Five expression vectors for production of recombinant DNA methyltransferases were constructed for this study. The version that expresses M.Ocy1ORF8430P, a putative ortholog of M.Spel, is shown. The others are similar in design but express M.Xbal, M.EcoRI, M.Pstl or M.Avalll instead. Each plasmid utilizes the low copy number p15A origin (pACYC) and confers resistance to spectinomycin and is thus compatible with pUC plasmids that impart resistance to ampicillin, chloramphenicol or kanamycin. The DNA methyltransferase expression vectors do not contain any of the restriction sites employed in BioBrick assembly protocols (EcoRI, Xbal, Spel or Pstl), so they will not produce restriction fragments that ligate to those that are desired. 


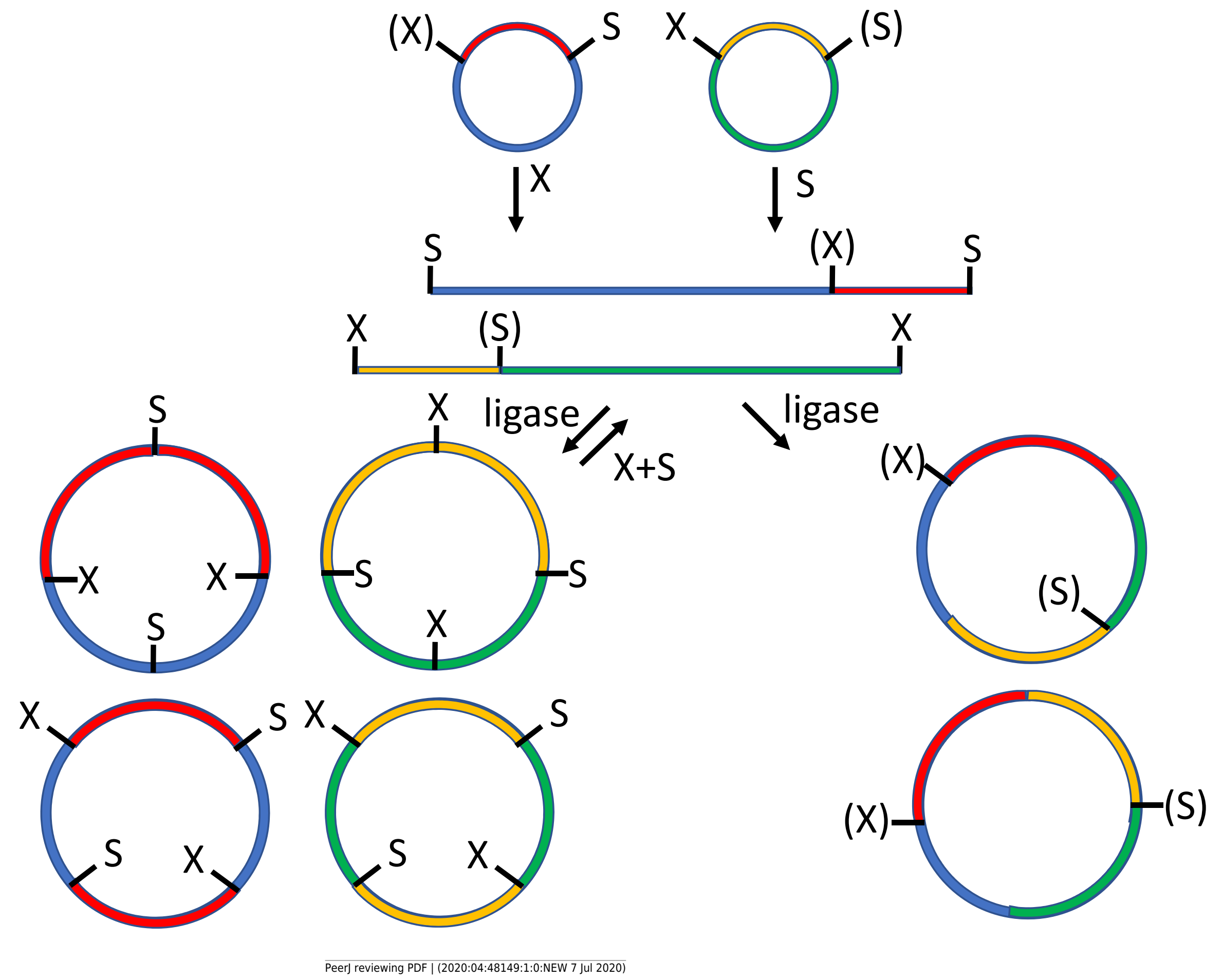


Figure 6

2RM assembly intermediates

Plasmids 1 (lacl-Ptac-lacO-pUC, methylated at its Xbal site) and 2 (tagRFP-pUC, methylated at its Spel site) were mixed and digested with both Xbal and Spel-HF. Each plasmid was protected from the action of one restriction enzyme and susceptible to the other (Figure 6).

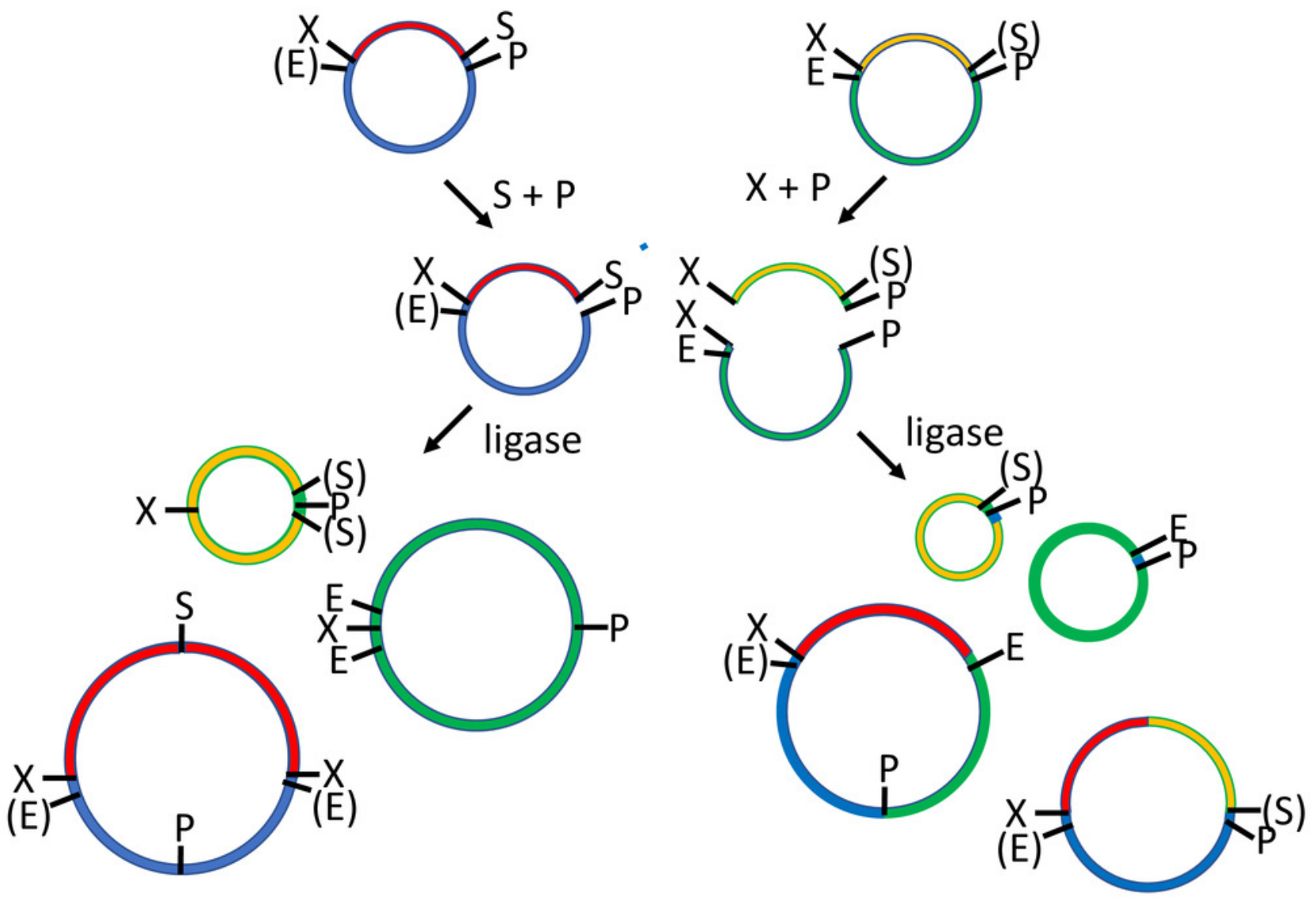

